\title{
GENERATORS OF REGULAR SEMIGROUPS
}

\author{
SHMUEL KANTOROVITZ \\ Bar-Ilan University 52900 Ramat-Gan, Israel \\ e-mail:kantor@math.biu.ac.il
}

(Received 23 October, 2006; revised 10 June, 2007; accepted 24 June, 2007)

\begin{abstract}
A regular semigroup (cf. [4, p. 38]) is a $C_{0}$-semigroup $T(\cdot)$ that has an extension as a holomorphic semigroup $W(\cdot)$ in the right halfplane $\mathbb{C}^{+}$, such that $\|W(\cdot)\|$ is bounded in the "unit rectangle" $Q:=(0,1] \times[-1,1]$. The important basic facts about a regular semigroup $T(\cdot)$ are: (i) it possesses a boundary group $U(\cdot)$, defined as the limit $\lim _{s \rightarrow 0+} W(s+i \cdot)$ in the strong operator topology; (ii) $U(\cdot)$ is a $C_{0}$-group, whose generator is $i A$, where $A$ denotes the generator of $T(\cdot)$; and (iii) $W(s+i t)=T(s) U(t)$ for all $s+i t \in \mathbb{C}^{+}$(cf. Theorems 17.9.1 and 17.9.2 in [3]). The following converse theorem is proved here. Let $A$ be the generator of a $C_{0}$-semigroup $T(\cdot)$. If $i A$ generates a $C_{0}$-group, $U(\cdot)$, then $T(\cdot)$ is a regular semigroup, and its holomorphic extension is given by (iii). This result is related to (but not included in) known results of Engel (cf. Theorem II.4.6 in [2]), Liu [7] and the author [6] for holomorphic extensions into arbitrary sectors, of $C_{0}$-semigroups that are bounded in every proper subsector. The method of proof is also different from the method used in these references. Criteria for generators of regular semigroups follow as easy corollaries.
\end{abstract}

2000 Mathematics Subject Classification. 47D03, 47D05

1. Introduction. The study of boundary values of holomorphic semigroups motivates the following definition.

DEFINITION 1.1 ( [4, p. 38]). A regular semigroup of operators on the Banach space $X$ is a $C_{0}$-semigroup $T(\cdot)$ on $X$, that has an extension as a holomorphic semigroup $W(\cdot)$ in the open halfplane $\mathbb{C}^{+}:=\{s+i t ; s>0, t \in \mathbb{R}\}$ such that $\|W(\cdot)\|$ is bounded in the "unit rectangle"

$$
Q:=\{s+i t ; 0<s \leq 1,-1 \leq t \leq 1\} .
$$

Recall that a holomorphic semigroup in the right halfplane is a function $W(\cdot): \mathbb{C}^{+} \cup$ $\{0\} \rightarrow B(X)$ with the following properties:

(a) $W(\cdot)$ is holomorphic in $\mathbb{C}^{+}$;

(b) $W(\cdot)$ is strongly continuous at 0 ; and

(c) $W(0)=I$ and $W(z+w)=W(z) W(w)$ for all $z, w \in \mathbb{C}^{+}$.

A regular semigroup $T(\cdot)$ is characterized by the existence of a (unique) holomorphic extension $W(\cdot)$ in $\mathbb{C}^{+}$, that possesses a boundary group $U(\cdot)$, defined as the limit

$$
U(t):=\lim _{s \rightarrow 0+} W(s+i t) \quad(t \in \mathbb{R})
$$

in the strong operator topology. 
The group $U(\cdot)$ is a $C_{0}$-group commuting with $W(\cdot)$, and

$$
W(s+i t)=T(s) U(t) \quad(s>0 ; t \in \mathbb{R}) .
$$

Furthermore, if $A$ denotes the (infinitesimal) generator of the regular semigroup $T(\cdot)$, then $i A$ is the generator of the associated boundary group $U(\cdot)$. Cf. [3, Theorems 17.9.1 and 17.9.2].

Conversely, we shall prove in Section 2 that if $T(\cdot)$ is a $C_{0}$-semigroup, whose generator $A$ is such that $i A$ generates a $C_{0}$-group, $U(\cdot)$, then $T(\cdot)$ is a regular semigroup. When this is the case, the unique holomorphic extension of $T(\cdot)$ to $\mathbb{C}^{+}$is given by (3).

As a consequence, we obtain conditions on an operator $A$ that are necessary and sufficient for it to be the generator of a regular semigroup.

For convenience, we use [5] as a reference for needed facts about semigroups.

We wish to thank the referee for his useful suggestions (especially for the remark following Theorem 2.1 and a simplification of the proof of Corollary 3.6).

\section{Characterization of regular semigroups.}

TheOREM 2.1. Let $T(\cdot)$ be a $C_{0}$-semigroup, and let $A$ be its generator. Then $T(\cdot)$ is a regular semigroup if and only if $i A$ is the generator of a $C_{0}$-group, $U(\cdot)$. In this case, the (unique) holomorphic extension of $T(\cdot)$ to $\mathbb{C}^{+}$is given by $W(s+i t)=T(s) U(t)$, $(s>0 ; t \in \mathbb{R})$.

(The associated boundary group is necessarily $U(\cdot)$.)

This result is not contained in [2, Theorem II.4.6], that concerns the holomorphic extension into a sector of a bounded $C_{0}$-semigroup, that is bounded in each proper subsector (cf. [2, Definition II.4.5]). Note the equivalent Condition (e) in [2, Theorem II.4.6] (" $A$ is sectorial"), that includes in particular the requirement that the resolvent set of $A$ contains a sector of half opening strictly greater than $\pi / 2$ (cf. [2, Definition II.4.1]), while no such requirement is made here.

Proof. We discussed the necessity part in Section 1. To prove the sufficiency part, suppose that $i A$ generates a $C_{0}$-group $U(\cdot)$. By [5, Theorem 1.1] applied to the $C_{0^{-}}$ semigroups $\{T(t) ; t \geq 0\},\{U(t) ; t \geq 0\}$, and $\{U(-t ; t \geq 0\}$, there exist constants $a, b \geq 0$ and $M, N \geq 1$ such that

$$
\|T(s)\| \leq M e^{a s} ; \quad\|U(t)\| \leq N e^{b|t|} \quad(s \geq 0 ; t \in \mathbb{R}) .
$$

We define $W(\cdot)$ on $\mathbb{C}^{+}$by (3).

Fix $x$ in the domain $D(A)$ of $A$, and consider the $X$-valued function on $\mathbb{C}^{+}$

$$
g(s+i t):=W(s+i t) A x=T(s) U(t) A x=T(s) A U(t) x \quad(s>0 ; t \in \mathbb{R}) .
$$

(Cf. [5, Theorem 1.2].)

Observe that $g$ is strongly continuous in $\mathbb{C}^{+}$, because if $s+i t, s^{\prime}+i t^{\prime} \in \mathbb{C}^{+}$, then

$$
\begin{aligned}
\| g(s & +i t)-g\left(s^{\prime}+i t^{\prime}\right)\|\leq\|\left[T(s)-T\left(s^{\prime}\right)\right][U(t) A x]\|+\| T\left(s^{\prime}\right)\left[U(t)-U\left(t^{\prime}\right)\right] A x \| \\
& \leq\left\|\left[T(s)-T\left(s^{\prime}\right)\right][U(t) A x]\right\|+M e^{a s^{\prime}}\left\|\left[U(t)-U\left(t^{\prime}\right)\right] A x\right\| \rightarrow 0
\end{aligned}
$$

when $s^{\prime}+i t^{\prime} \rightarrow s+i t$, by (4) and the strong continuity of $T(\cdot)$ and $U(\cdot)$ (cf. [5, Theorem 1.1]). 
Next, since $A$ generates $T(\cdot)$ and $i A$ generates $U(\cdot)$, it follows from the definition (3) of $W(\cdot)$ and [5, Theorem 1.2] that for all $s+i t \in \mathbb{C}^{+}$

$$
\frac{\partial}{\partial s} W(s+i t) x=T(s) A U(t) x=-i T(s)(i A) U(t) x=-i \frac{\partial}{\partial t} W(s+i t) x .
$$

Thus, for each $x \in D(A)$ and $x^{*} \in X^{*}$, the complex valued function $x^{*} W(\cdot) x$ satisfies the Cauchy-Riemann equation and has continuous partial derivatives (by our observation on the function $g$ ) in $\mathbb{C}^{+}$. Therefore, by (the classical) Theorem 11.2 in $[\mathbf{8}], x^{*} W(\cdot) x$ is holomorphic in $\mathbb{C}^{+}$for all $x \in D(A)$ and $x^{*} \in X^{*}$.

Fix $x^{*} \in X^{*}$, and let $0 \neq x \in X$ be arbitrary. Since $D(A)$ is dense in $X$ (cf. [5, Theorem 1.2]), we may choose a sequence $\left\{x_{n}\right\} \subset D(A)$ such that $x_{n} \rightarrow x$ in $X$ and $\left\|x_{n}\right\| \leq 2\|x\|$ for all $n \in \mathbb{N}$.

Let $H$ be any compact subset of $\mathbb{C}^{+}$, and set

$$
\sigma=\sigma(H):=\max _{z \in H} \Re z ; \quad \tau=\tau(H):=\max _{z \in H}|\Im z| .
$$

Then for all $s+i t \in H$ and $n \in \mathbb{N}$,

$$
\left|x^{*} W(s+i t) x_{n}\right| \leq 2 M N e^{a \sigma+b \tau}|| x^{*}||\|x\|,
$$

that is, $\left\{x^{*} W(\cdot) x_{n} ; n \in \mathbb{N}\right\}$ is a family of holomorphic functions in $\mathbb{C}^{+}$, uniformly bounded on each compact subset of $\mathbb{C}^{+}$. It is therefore a normal family (cf. [8, Theorem 14.6]). Let then $\left\{x^{*} W(\cdot) x_{n_{k}}\right\}$ be a subsequence converging uniformly on compact subsets of $\mathbb{C}^{+}$. The limit function, $x^{*} W(\cdot) x$, is then holomorphic in $\mathbb{C}^{+}$(cf. [8, Theorem 10.27])). Since this is true for all $x \in X$ and $x^{*} \in X^{*}$, it follows from [3, Theorem 3.10.1] that the operator valued function $W(\cdot)$ is holomorphic in $\mathbb{C}^{+}$. For all $s>0$, we have $W(s)=T(s) U(0)=T(s)$ (by definition), so that $W(\cdot)$ is indeed a holomorphic extension of $T(\cdot)$ to $\mathbb{C}^{+}$.

For any $w=u+i v$ with $u>0$ and $v \in \mathbb{R}$, the functions $W(\cdot) W(w)$ and $W(\cdot+w)$ are holomorphic in $\mathbb{C}^{+}$and agree on $(0, \infty)$, since for all $s>0$,

$$
W(s) W(w)=T(s) T(u) U(v)=T(s+u) U(v)=W(s+u+i v)=W(s+w) .
$$

Therefore $W(z) W(w)=W(z+w)$ for all $z, w \in \mathbb{C}^{+}$.

The strong continuity at 0 of $W(\cdot)$ follows from (4) and the $C_{0}$-property of $T(\cdot)$ and $U(\cdot)$. Indeed, for all $x \in X, s>0$, and $t \in \mathbb{R}$, we have

$$
\begin{gathered}
\|W(s+i t) x-x\| \leq\|T(s)[U(t) x-x]\|+\|T(s) x-x\| \\
\leq M e^{a s}\|U(t) x-x\|+\|T(s) x-x\| \rightarrow 0
\end{gathered}
$$

as $s \rightarrow 0+$ and $t \rightarrow 0$.

We conclude that $W(\cdot)$ is a holomorphic semigroup extending $T(\cdot)$ to $\mathbb{C}^{+}$. It is clearly bounded on the "unit rectangle" $Q$, since by (3) and (4), for all $z=s+i t \in Q$,

$$
\|W(z)\| \leq\|T(s)\|\|U(t)\| \leq M N e^{a+b}<\infty .
$$

This shows that $T(\cdot)$ is indeed a regular semigroup (with the said extension ).

REMARK. The normal family argument in the proof could be replaced by an application of Morera's theorem. Indeed, fix $x \in X, x^{*} \in X^{*}$, and let $x_{n} \in D(A)$ be such that 
$x_{n} \rightarrow x$. Then $x^{*} W(\cdot) x_{n}$ are holomorphic in $\mathbb{C}^{+}$(by the first part of the proof, since $x_{n} \in D(A)$ ), and $x^{*} W(\cdot) x_{n} \rightarrow x^{*} W(\cdot) x$ pointwise. Let $H$ be any triangular path in $\mathbb{C}^{+}$, and let $\sigma=\sigma(H)$ and $\tau=\tau(H)$ as in (7). Then

$$
\left\|x^{*} W(\cdot) x\right\| \leq M N e^{a \sigma+b \tau}\left\|x^{*}\right\|\|x\|
$$

on $H$. By dominated convergence and analyticity of $x^{*} W(\cdot) x_{n}$ in $\mathbb{C}^{+}$,

$$
\int_{H} x^{*} W(z) x d z=\lim _{n} \int_{H} x^{*} W(z) x_{n} d z=0 .
$$

As in the argument showing the continuity of $g$ in the proof above, it follows from (4) and the $C_{0}$ property of $T(\cdot)$ and $U(\cdot)$ that $x^{*} W(\cdot) x$ is continuous in $\mathbb{C}^{+}$, and Morera's theorem now implies its analyticity, as desired.

The theorem may be restated as a solution of the extension problem of a given $C_{0}$-group $U(\cdot)$ to a holomorphic semigroup in $\mathbb{C}^{+}$, whose boundary group is the group $U(\cdot)$. (Cf. [3, Theorem 17.10.1] for a more technical solution).

TheOREM 2.2. Let $U(\cdot)$ be a $C_{0}$-group, and let $i A$ be its generator. Then $U(\cdot)$ is the boundary group associated with a regular semigroup if and only if $A$ generates a $C_{0}$-semigroup, $T(\cdot)$.

In this case, $T(\cdot)$ is the unique regular semigroup with associated boundary group $U(\cdot)$, and the unique holomorphic extension of $U(\cdot)$ to $\mathbb{C}^{+}$is given by (3).

3. Characterization of generators of regular semigroups. The characterization of a regular semigroup provided by Theorem 2.1, combined with various versions of the Hille-Yosida theorem (cf. [5, Theorem 1.17 and Corollary 1.18]), yield easily to characterizations of generators of regular semigroups.

Corollary 3.1. Let $A$ be an operator on $X$ with domain $D(A)$. Then $A$ generates a regular semigroup if and only if the following conditions (a)-(c) are satisfied:

(a) $D(A)$ is dense in $X$;

(b) the resolvent set of $A$ contains the rays $(a, \infty)$ and $\pm i(b, \infty)$, for some $a, b \geq 0$;

(c) $\sup _{s>a ; n \in \mathbb{N}}\left\|[(s-a) R(s ; A)]^{n}\right\|<\infty$; and $\sup _{t>b ; n \in \mathbb{N}} \|\left[(t-b) R(( \pm i t ; A)]^{n} \|<\right.$ $\infty$.

Proof. If $A$ generates a regular semigroup, then $i A$ generates a $C_{0}$-group, and conditions (a)-(c) follow from the necessity part of the Hille-Yosida theorem for the generators $A$ and $i A$ and the relation

$$
R(\lambda ; i A)=-i R(-i \lambda ; A)
$$

Conversely, suppose $A$ satisfies Conditions (a)-(c). Since $A$ has a non-empty resolvent set (by (b)), it is a closed operator. Therefore, by (a), (b), and the first condition in (c), it follows from the Hille-Yosida theorem ([5, Theorem 1.17]) that $A$ generates a $C_{0}$-semigroup. By (8), the second condition in (c) may be written in the form

$$
\left.\sup _{t>b ; n \in \mathbb{N}} \|(t-b) R( \pm t ; i A)\right]^{n} \|<\infty .
$$


Together with (a) and (b), this implies that $i A$ generates a $C_{0}$-group (cf. [5, Theorem 1.39]). By Theorem 2.1, we conclude that $A$ generates a regular semigroup.

COROllary 3.2. Let $T(\cdot)$ be a $C_{0}$-semigroup of contractions, and let $A$ be its generator. Then $T(\cdot)$ extends to a holomorphic semigroup of contractions in $\mathbb{C}^{+}$if and only if $i$ generates a $C_{0}$-group of contractions.

(As observed before, since $A$ is not necessarily "sectorial", this corollary does not follow from [2, Theorem II.4.6]; cf. Condition (e) in this theorem and [2, Definition II.4.1].)

The sufficient (and necessary) condition in Corollary 3.2 should be compared to the condition given in [5, Theorem 1.54] (see also [6]). There, in the general case of holomorphic extensions to arbitrary sectors

$$
S_{\theta}:=\left\{z=r e^{i \phi} ; r>0,-\theta<\phi<\theta\right\}
$$

with $0<\theta \leq \pi / 2$, the (necessary and) sufficient condition is that $e^{i \alpha} A$ generate a $C_{0}$-semigroup of contractions for all $\alpha \in(-\theta, \theta)$. Here, in the particular case of holomorphic extension to the right halfplane (case $\theta=\pi / 2$ ), we obtained the preceding (necessary and) sufficient condition involving only the endpoint values $\alpha= \pm \pi / 2$ (namely, that $\pm i A$ generate $C_{0}$-semigroups of contractions).

In Liu [7], $A$ is even assumed to have a bounded everywhere defined inverse, a condition that is not needed here.

Proof. If $T(\cdot)$ extends to a holomorphic semigroup of contractions in $\mathbb{C}^{+}$, it is trivially regular, and therefore $i A$ generates the associated boundary group, that is necessarily a $C_{0}$-group of contractions. Conversely, if $i A$ generates a $C_{0}$-group of contractions $U(\cdot)$, then by Theorem $2.1, T(\cdot)$ is regular, and its unique extension as a holomorphic semigroup in $\mathbb{C}^{+}$is $W(s+i t)=T(s) U(t)$, that consists clearly of contractions.

COROLlaRY 3.3. The operator A generates a holomorphic contraction semigroup in $\mathbb{C}^{+}$if and only if the following conditions (a) and (b) are satisfied:

(a) $D(A)$ is dense in $X$;

(b) $s R(s ; A)$ and $t R( \pm i t ; A)$ exist and are contractions for all $s, t>0$.

Proof. This follows from [5, Corollary 1.18], Corollary 3.2, and (8).

The next two corollaries deal with perturbations of regular semigroups generators. They follow almost trivially from Theorem 2.1 and known perturbation theorems for $C_{0}$-semigroups, but do not seem to be found in the literature.

Corollary 3.4. Let $A$ generate a regular semigroup, and let $B \in B(X)$. Then $A+B$ generates a regular semigroup.

Proof. By a special case of the Hille-Phillips perturbation theorem (cf. [5, Theorem 1.38]) and the necessity part of Theorem 2.1, the perturbations $A+B$ and $i(A+$ $B)=(i A)+(i B)$ generate a $C_{0}$-semigroup and a $C_{0}$-group respectively. Therefore $A+B$ generates a regular semigroup, by the sufficiency part of Theorem 2.1. 
In the special case when $A$ generates a holomorphic $C_{0}$-semigroup of contractions in $\mathbb{C}^{+}$(that is, a $C_{0}$-semigroup of contractions $T(\cdot)$ on $[0, \infty)$ that extends to a holomorphic semigroup of contractions $W(\cdot)$ in $\mathbb{C}^{+}$), we may apply [5, Theorem 1.30$]$ to get the following perturbation result. Recall that the numerical range of an operator $B$ is the set

$$
v(B):=\left\{x^{*} B x ; x \in D(B), x^{*} \in X^{*},\|x\|=\left\|x^{*}\right\|=x^{*} x=1\right\} .
$$

COROLlaRY 3.5. Let A generate a holomorphic $C_{0}$-semigroup of contractions in $\mathbb{C}^{+}$. Let $B$ be an operator satisfying the following conditions (a) and (b):

(a) $v(B) \subset(-\infty, 0]$;

(b) $D(A) \subset D(B)$ and there exist constants $0 \leq a<1$ and $b \geq 0$ such that

$$
\|B x\| \leq a\|A x\|+b\|x\|
$$

for all $x \in D(A)$.

Then $A+B$ generates a holomorphic $C_{0}$-semigroup of contractions in $\mathbb{C}^{+}$.

Proof. By [5, Theorem 1.30], $A+B$ generates a $C_{0}$-semigroup of contractions. By Corollary 3.2, $\pm i A$ generate $C_{0}$-semigroups of contractions. By Condition (a),

$$
\Re v( \pm i B)=\mp \Im v(B)=0,
$$

and therefore $i B$ and $-i B$ are trivially dissipative. They are also $i A$-bounded and $-i A$ bounded (respectively), with $i A$-bound ( $-i A$-bound, respectively) smaller than 1 (by Condition (b)). Consequently, by [5, Theorem 1.30], the operators $i(A+B)$ and $-i(A+$ $B$ ) generate $C_{0}$-semigroups of contractions. We now conclude from Corollary 3.2 that $A+B$ generates a holomorphic $C_{0}$-semigroup of contractions.

Our last corollary gives a growth estimate of a regular semigroup $T(\cdot)$ in terms of any fixed value of the norm $\|T(c)\|$ (the result may be new).

COROLlARY 3.6. Let $T(\cdot)$ be a regular semigroup. Let $U(\cdot)$ be its boundary group, and let $b \geq 0$ and $N \geq 1$ be constants such that $\|U(t)\| \leq N e^{b|t|}$ for all $t \in \mathbb{R}$ (cf. (4)). Fix $c>0$. Then

$$
\|T(s)\| \leq N e^{b c / 2}\|T(c)\|^{s / c}
$$

for all $s>0$.

Proof. It suffices to prove the estimate for $c=1$, since the general case follows from this special case applied to the regular semigroup $T^{\prime}(s):=T(c s)$. Since the estimate is trivial for $s \in \mathbb{N}$ (if $s=n \in \mathbb{N}$, then $\|T(s)\|=\left\|T(1)^{n}\right\| \leq\|T(1)\|^{n} \leq N e^{b / 2}\|T(1)\|^{s}$, since $N \geq 1$ and $b \geq 0$ ), it suffices to consider non-integral $s>0$. This will follow in turn from the special case $0<s<1$, because writing $s=n+t$ with $n$ a non-negative integer and $0<t<1$, we get (from the said special case)

$$
\begin{aligned}
& \|T(s)\|=\left\|T(1)^{n} T(t)\right\| \leq\|T(1)\|^{n}\|T(t)\| \\
& \leq N e^{b / 2}\|T(1)\|^{t}\|T(1)\|^{n}=N e^{b / 2}\|T(1)\|^{s} .
\end{aligned}
$$

By Theorem 2.1, the holomorphic extension of $T(\cdot)$ to $\mathbb{C}^{+}$is given by $W(s+i t)=$ $T(s) U(t)$. Consider the operator-valued continuous function $\Phi(z)=e^{b z^{2}} W(z)$ on $\overline{\mathbb{C}^{+}}$. 
It is holomorphic in $\mathbb{C}^{+}$, and

$$
\begin{aligned}
\|\Phi(s+i t)\| & =e^{b\left(s^{2}-t^{2}\right)}\|T(s) U(t)\| \leq N \exp \left(b\left[s^{2}-t^{2}+|t|\right]\right)\|T(s)\| \\
& \leq N \exp \left(b\left[s^{2}+1 / 4\right]\right)\|T(s)\|
\end{aligned}
$$

(because $\left.-t^{2}+|t|=|t|(1-|t|) \leq 1 / 4\right)$. By (9), $\Phi$ is bounded in the vertical strip $\{s+i t ; 0 \leq s \leq 1, t \in \mathbb{R}\}$. Also, for all $t \in \mathbb{R}$,

$$
\|\Phi(i t)\| \leq N e^{b / 4} ; \quad\|\Phi(1+i t)\| \leq N e^{b(1+1 / 4)}\|T(1)\| .
$$

If $s \in(0,1)$, write $s$ as the convex combination $s=p .0+(1-p) .1=1-p$ with $p \in$ $(0,1)$. By the "Three Lines theorem" for operator-valued holomorphic functions (cf. [1, Theorem VI.10.3]), it follows from (10) that for all $s \in(0,1)$ and $t \in \mathbb{R}$,

$$
\|\Phi(s+i t)\| \leq N e^{b / 4} e^{b s}\|T(1)\|^{s} .
$$

Taking $t=0$, it follows from (9) that for all $s \in(0,1)$

$$
\|T(s)\| \leq N e^{b / 4} e^{b s(1-s)}\|T(1)\|^{s} \leq N e^{b / 2}\|T(1)\|^{s}
$$

(because $s(1-s) \leq 1 / 4$ for $s \in(0,1)$ ).

\section{REFERENCES}

1. N. Dunford and J. T. Schwartz, Linear operators, Volume 1 (Interscience Publishers, New York, 1958).

2. K. Engel and R. Nagel, One parameter semigroups for linear evolution equations, Graduate Texts in Mathematics (Springer-Verlag, 2000).

3. E. Hille and R. S. Phillips, Functional analysis and semigroups, Amer. Math. Soc. Coll. Publ. 31 (Providence, R.I., 1957).

4. S. Kantorovitz, Spectral theory of Banach space operators, Lecture Notes in Math., Vol. 1012 (Springer-Verlag, 1983).

5. S. Kantorovitz, Semigroups of operators and spectral theory, Pitman Research Notes in Math., Vol. 330 (Longman, New York, 1995).

6. S. Kantorovitz, On Liu's analyticity criterion for semigroups, Semigroup Forum $\mathbf{5 3}$ (1996), 262-265.

7. Y. Liu, An equivalent condition for analytic $C_{0}$-semigroups, J. Math. Anal. Appl. 180 (1993), 71-78.

8. W. Rudin, Real and complex analysis (McGraw-Hill, 1966). 